\title{
Metformin inhibits growth of eutopic stromal cells from adenomyotic endometrium via AMPK activation and subsequent inhibition of AKT phosphorylation: a possible role in the treatment of adenomyosis
}

\author{
Jing Xue, Hui Zhang, Wei Liu, Ming Liu, Min Shi, Zeqing Wen and Changzhong Li \\ Department of Obstetrics and Gynecology, Provincial Hospital Affiliated to Shandong University, \\ 324 Jingwu Road, Jinan, Shandong 250021, People's Republic of China
}

Correspondence should be addressed to C Li; Email: lichangzhong@hotmail.com

\begin{abstract}
Adenomyosis is a finding that is associated with dysmenorrhea and heavy menstrual bleeding, associated with PI3K/AKT signaling overactivity. To investigate the effect of metformin on the growth of eutopic endometrial stromal cells (ESCs) from patients with adenomyosis and to explore the involvement of AMP-activated protein kinase (AMPK) and PI3K/AKT pathways. Primary cultures of human ESCs were derived from normal endometrium (normal endometrial stromal cells (N-ESCs)) and adenomyotic eutopic endometrium (adenomyotic endometrial stroma cells (A-ESCs)). Expression of AMPK was determined using immunocytochemistry and western blot analysis. 3-(4, 5-Dimethylthiazol-2-yl)-2, 5-diphenyltetrazolium bromide assays were used to determine the effects of metformin and compound C on ESCs and also to detect growth and proliferation of ESCs. AMPK and PI3K/AKT signaling was determined by western blotting. A-ECSs exhibited greater AMPK expression than N-ESCs. Metformin inhibited proliferation of ESCs in a concentration-dependent manner. The IC $\mathrm{IC}_{50}$ was $2.45 \mathrm{mmol} / \mathrm{I}$ for A-ESCs and $7.87 \mathrm{mmol} / \mathrm{I}$ for N-ESCs. Metformin increased AMPK activation levels (p-AMPK/AMPK) by 2.0 \pm 0.3-fold in A-ESCs, 2.3-fold in A-ESCs from the secretory phase, and 1.6-fold in the proliferation phase. The average reduction ratio of $17 \beta$-estradiol on A-ESCs was $2.1 \pm 0.8$-fold in proliferative phase and $2.5 \pm 0.5$-fold in secretory phase relative to the equivalent groups not treated with $17 \beta$-estradiol. The inhibitory effects of metformin on AKT activation (p-AKT/AKT) were more pronounced in A-ESCs from the secretory phase (3.2-fold inhibition vs control) than in those from the proliferation phase (2.3-fold inhibition vs control). Compound C, a selective AMPK inhibitor, abolished the effects of metformin on cell growth and PI3K/AKT signaling. Metformin inhibits cell growth via AMPK activation and subsequent inhibition of PI3K/AKT signaling in A-ESCs, particularly during the secretory phase, suggesting a greater effect of metformin on A-ESCs from secretory phase.

Reproduction (2013) 146 397-406
\end{abstract}

\section{Introduction}

Women with dysmenorrhea, chronic pelvic pain, and heavy menstrual bleeding present a diagnostic and therapeutic challenge. Adenomyosis is a commonly recognized cause of the above-mentioned symptoms. Adenomyosis is characterized by the presence of ectopic endometrial tissue in the myometrium, with adjacent smooth muscle hyperplasia (Devlieger et al. 2003, Harvey \& Warwick 2010). Adenomyosis is usually found in multiparous, pre-menopausal women older than 30 years, particularly those with previous cesarean section or other uterine surgery (Takeuchi \& Matsuzaki 2011).

Adenomyosis is usually diagnosed by histological evaluation of a hysterectomy specimen showing invasion of endometrial tissue into the myometrium (Brown et al. 1991). Ultrasound or magnetic resonance imaging
(MRI) may aid in the nonsurgical diagnosis of adenomyosis, with MRI having $78-88 \%$ sensitivity and $67-93 \%$ specificity (Ascher et al. 1994, Reinhold et al. 1996, Atri et al. 2000, Dueholm et al. 2001, Bazot et al. 2001).

Adenomyosis is non-neoplastic and hence is not associated with significant mortality. It does, however, cause substantial morbidity and reduced quality of life for the many individuals it affects. Patients typically present with menorrhagia, dysmenorrhea, metrorrhagia, and dyspareunia, which may be a cause of reduced fertility (Devlieger et al. 2003, Takeuchi \& Matsuzaki 2011). Surgical hysterectomy remains the only definitive treatment (Brown et al. 1991, Devlieger et al. 2003). Surgery is usually physiologically and psychologically traumatic for patients of reproductive age, highlighting the need to develop novel and effective treatments. 
There is substantial evidence to indicate that adenomyosis and endometriosis are influenced and promoted by the effects of estrogen (Cakmak et al. 2009). Indeed, the estrogen agonist tamoxifen has been successfully used to generate mouse models of adenomyosis (Li et al. 2011). Leyendecker et al. (2009) have proposed a tissue injury and repair theory of adenomyosis, in which microtrauma at the endometrial-myometrial junction causes local hyperestrogenism and enhanced peristaltic activity within the zone of the endomyometrial junction. Estrogen is known to act through the MAPK and PI3K/AKT/mTOR pathways, raising the possibility that targeting the $\mathrm{PI} 3 \mathrm{~K}$ pathway may provide a novel approach for the treatment of these conditions (Cakmak et al. 2009, Leyendecker et al. 2009, Makker et al. 2012). In support of this possibility, other workers have reported that overactivation of $\mathrm{PI} 3 \mathrm{~K}$ signaling occurs in eutopic and ectopic endometria (Zhang et al. 2010).

AMP-activated protein kinase (AMPK) is a heterotrimeric serine/threonine protein kinase complex, comprising a catalytic $\alpha$-subunit and regulatory $\beta$ - and $\gamma$-subunits (Hardie 2011). AMPK signaling regulates energy metabolism and is activated in response to cellular stresses that deplete cellular energy levels and increase the AMP:ATP ratio (Carling 2004). Once activated, AMPK restores cellular energy levels by stimulating catabolic pathways, such as glucose uptake, glycolysis, and fatty acid oxidation, and by halting ATP-consuming processes, such as fatty acid, cholesterol, and protein synthesis (Lim et al. 2009). AMPK signaling is also linked to the PI3K pathway (Tao et al. 2010), which is an important regulator of cell survival (Chen et al. 2010). AMPK signaling has, therefore, been investigated as a potential therapeutic target for the treatment of diseases with aberrantly activated PI3K signaling, such as carcinomas (van Veelen et al. 2011, Carling et al. 2012, Martelli et al. 2012). To date, however, to our knowledge no studies have investigated whether AMPK may play a role in adenomyosis.

Metformin is a biguanide drug that is widely used as a first-line pharmacological treatment in patients with type 2 diabetes (Cantrell et al. 2010). Metformin exerts its effects through the activation of AMPK in muscle, adipose tissue, and liver (Towler \& Hardie 2007). However, little is known about its effects on endometrial tissues, and whether these effects may include actions via PI3K/AKT signaling that would be beneficial for the treatment of adenomyosis.

In this in vitro study, we explored the role of AMPK signaling in the adenomyotic eutopic endometrium and investigated the effects of activation and inhibition of AMPK signaling on the proliferation of eutopic stromal cells. We also assessed the role of PI3K/AKT signaling and investigated the possible value and mechanism of metformin in the treatment of adenomyosis.

\section{Materials and methods}

\section{Reagents and antibodies}

Collagenase IA, trypsin, metformin, and $17 \beta$-estradiol $\left(E_{2}\right)$ were obtained from Sigma Corporation and compound $C$ from Merck Serono. Penicillin and DMEM:nutrient mixture F-12 (DMEM/F12) (1:1) were purchased from HyClone Corporation (Beijing, China). Charcoal-stripped fetal bovine serum (FBS), in which hormones and certain growth factors had been excluded, was obtained from Gibco (Invitrogen). Rabbit primary antibodies against human AMPK, p-AMPK (Thr172), AKT, p-AKT (Ser473), and $\beta$-actin were obtained from Cell Signaling Technology (Danvers, MA, USA). Mouse antibodies against human vimentin and cytokeratin were obtained from Cell Signaling Technology. Horseradish peroxidase (HRP)conjugated goat anti-rabbit secondary antibodies and diaminobenzidine $(\mathrm{DAB})$ staining kits were purchased from Jingmei Biotech Co. Ltd. (Shanxi, China). 3-(4, 5-Dimethylthiazol-2-yl)-2, 5-diphenyltetrazolium bromide (MTT), RIPA buffer, and the Mammalian Cell Extraction Kit were purchased from BioVision (Milpitas, CA, USA). The ECL Plus Western Blotting Detection System was obtained from Amersham Biosciences and the Bradford protein assay kit from Bio-Rad.

Compound $\mathrm{C}$ was dissolved in DMSO at a stock concentration of $10 \mathrm{mmol} / \mathrm{l}$ and stored at $4{ }^{\circ} \mathrm{C}$. A final compound $\mathrm{C}$ concentration of $40 \mu \mathrm{mol} / \mathrm{l}$ was used in cell cultures, and the final concentration of DMSO was $<0.1 \%$. The same volume/concentration of DMSO was used in control experiments. Metformin was dissolved in double-distilled water at a stock concentration of $10 \mathrm{mmol} / \mathrm{l}$ and stored at $4{ }^{\circ} \mathrm{C}$. $\mathrm{E}_{2}$ was dissolved in absolute ethyl alcohol at a stock concentration of $10 \mathrm{mmol} / \mathrm{l}$ and stored at $4{ }^{\circ} \mathrm{C}$. A final $\mathrm{E}_{2}$ concentration of $10 \mathrm{nM}$ was used in cell cultures, such that the final concentration of absolute ethyl alcohol was $<0.1 \%$. The same concentration/ volume of DMSO were used in control experiments.

\section{Subjects and specimens}

The experiments were performed using endometrial biopsies of eutopic endometrium taken from 34 patients with adenomyosis, $42.1 \pm 2.4$ years of age. Fifteen tissue samples were taken during the proliferative phase $(42.7 \pm 1.9$ years $)$ and 19 during the secretory phase $(41.3 \pm 2.4$ years $)$. The diagnosis was confirmed by pathological examination, which excluded the presence of other gynecological diseases. Endometrial biopsies taken from 19 women of reproductive age (mean: $43.7 \pm 3.2$ years) without endometriosis undergoing bilateral tubal ligation were used as a control group. None of the participants had received hormonal therapy during the 6 months prior to surgery.

The phase of the menstrual cycle (proliferative phase or secretory phase) was determined on the basis of the time of the last menstruation, $\mathrm{E}_{2}$ and progesterone levels of participants before surgery, findings during surgery, and the histological pattern of the endometrium (Noyes et al. 1975).

All participants provided written informed consent prior to biopsy; the use of human tissues was approved by the Institutional Review Board of Shandong Provincial Hospital Affiliated to Shandong University (China). 


\section{Purification of endometrial stromal cells}

The isolation and culture of endometrial cells were based on methods described in previous studies (Sugawara et al. 1997, Yang et al. 2007), with slight modifications. Briefly, specimens obtained during surgery were placed immediately in ice-cold sterile PBS and transported to the laboratory. Tissues were washed two times with sterile PBS, then homogenized, and incubated with $0.25 \%$ collagenase type IA, in a shaking water bath for $1 \mathrm{~h}$ at $37^{\circ} \mathrm{C}$. The collagenase activity was terminated by the addition of three volumes of pre-warmed PBS.

The cell suspension was sequentially filtered through a $154 \mu \mathrm{m}$ monofilament nylon mesh and then through a $38.5 \mu \mathrm{m}$ monofilament nylon mesh. The resulting cell suspension was collected and centrifuged at $110 \mathrm{~g}$ for 10 min to obtain stromal cells. The pellet was re-suspended in DMEM/F12 (1:1) medium containing $10 \%$ FBS and incubated in cell culture dishes for $2 \mathrm{~h}$ at $37{ }^{\circ} \mathrm{C}$ in $95 \%$ air and $5 \% \mathrm{CO}_{2}$. The medium was then replaced with fresh medium; non-attached cells were discarded and the attached stromal cells were cultured further.

The culture medium was changed every 2-3 days. Normal endometrial stromal cells (N-ESCs) and adenomyotic eutopic ESCs (A-ESCs) were isolated and cultured in DMEM/F12 (1:1) medium, containing $10 \%$ charcoal-stripped $\mathrm{FBS}$, penicillin $(100 \mathrm{U} / \mathrm{ml})$, and streptomycin $(100 \mathrm{U} / \mathrm{ml})$ at $37^{\circ} \mathrm{C}$ in a humidified environment with $5 \% \mathrm{CO}_{2}$ in air. The cultured endometrial cells were characterized by immunocytochemical staining for vimentin and cytokeratin (Bruse et al. 2005); meanwhile, the purity of ESCs was tested.

\section{Immunocytochemistry}

The cultured ESCs on coverslips were washed twice with PBS and fixed in 4\% paraformaldehyde ( $\mathrm{pH} 7.0)$ for more than $24 \mathrm{~h}$. The cells were then washed in PBS, blocked with $10 \%$ normal goat serum for $30 \mathrm{~min}$, and incubated overnight with rabbit anti-human-AMPK primary antibody (diluted 1:500 in PBS) and with mouse antibodies against human vimentin and cytokeratin at $4{ }^{\circ} \mathrm{C}$. HRP-conjugated goat anti-rabbit IgG and HRP-conjugated goat anti-mouse $\operatorname{lgG}$ were used as secondary antibodies. HRP activity was detected using DAB tetrahydrochloride. Slides were counterstained with hematoxylin before mounting. Slides incubated with PBS instead of primary antibody were used as negative controls, and immunostaining with rabbit anti-human- $\beta$-actin antibody (at the same dilution) was used as a negative control for nuclear staining.

The immunocytochemical score was evaluated on the basis of the percentage of positively stained ESCs ( 0 , no staining; $1,1-10 \%$ positively staining cells; $2,11-50 \%$ positively staining cells; $3,51-80 \%$ positively staining cells; and 4, 81-100\% positively staining cells). The immunoreactivity was shown as a staining intensity score. Staining intensity was scored as follows: 0 , negative; 1 , weak staining; 2 , moderate staining; and 3, strong staining.

For each sample, two slides were assessed independently by two independent observers. Evaluation of slides for immunostaining was undertaken in a blinded fashion, without any knowledge of the clinical or pathological data.

\section{Cell proliferation assays}

The effect of metformin on cell proliferation was examined by the MTT assay. ESCs were plated in 96-well plates at a concentration of $1 \times 10^{3}$ cells/well. After attachment, cells were treated with different doses of metformin/compound $\mathrm{C}$ for $0 \mathrm{~min}, 15 \mathrm{~min}, 1 \mathrm{~h}$, and $24 \mathrm{~h}$. MTT assays were performed as described previously (Towler \& Hardie 2007). In brief, MTT $(5 \mathrm{mg} / \mathrm{ml})$ was added to the 96-well plates at a volume of $10 \mu \mathrm{l} /$ well, and the plates were incubated for $4 \mathrm{~h}$. The MTT reaction was terminated by removal of the culture medium containing MTT, and $100 \mu \mathrm{l}$ DMSO per well were added and incubated at RT on a shaker for 10 min to ensure that the crystals had dissolved sufficiently. Absorbance values were measured at $595 \mathrm{~nm}$. Cell proliferation (percentage of control) was calculated as follows: absorbance (experimental group)/absorbance (control group). Cell proliferation inhibition (percentage of control) was calculated as follows: $100 \%$-cell proliferation (percentage of control). Each experiment was performed in duplicate and repeated six times to assess result consistency.

\section{Western blotting}

ESCs were washed twice with ice-cold PBS. Cell lysates were prepared by incubation in RIPA buffer ( $1 \%$ NP40, 0.5 sodium deoxycholate, and $0.1 \%$ SDS) for $30 \mathrm{~min}$ on ice followed by centrifugation at 120000 r.p.m. (approximately $15000 \mathrm{~g}$ ) for $20 \mathrm{~min}$. The protein concentration of the extracts was determined using a Bradford protein assay kit. Equal amounts of protein $(30 \mu \mathrm{g})$ were separated by electrophoresis on a 10\% SDS-PAGE and transferred onto a PVDF membrane (Hybond-P PVDF membrane; Amersham). The membrane was blocked with $5 \%$ nonfat dry milk in Tris-buffered saline with Tween 20 for $1 \mathrm{~h}$ and incubated overnight with a 1:1000 diluted primary antibody at $4{ }^{\circ} \mathrm{C}$. The membrane was then washed and incubated for $1 \mathrm{~h}$ with a peroxidase-conjugated secondary antibody (diluted 1:10 000).

Antibody binding was detected using an ECL detection system (GE Healthcare, Princeton, NJ, USA). Western blotting densitometric analysis was performed using an Alphalmager 2200 gel documentation system with image analysis software. The band densities for AMPK and p-AMPK as well as Akt and $p$-Akt were normalized to that of $\beta$-actin. Each experiment was repeated in triplicate.

\section{Statistical analysis}

Statistical analysis was performed using SPSS version 17.0 Software (SPSS, Inc.). Data were expressed as the mean$\mathrm{s} \pm$ s.E.M.S. Comparisons between groups were performed using Student's $t$-test or one-way ANOVA followed by a Tukey's post-hoc test (for comparisons between treated and control ESCs and between groups in the cell proliferation experiments). For the analyses of experiments with three or four replicates, we used a non-parametric method. The data were shown as the median \pm range. Values of $P<0.05$ were considered statistically significant. 


\section{Results}

\section{Verification for the purity of ESCs}

Immunocytochemical staining for vimentin and cytokeratin was performed to test whether the purified cells were ESCs. As shown in Fig. 1, the purified cells were positive for vimentin and negative for cytokeratin, confirming that these cells were indeed ESCs. The purity of the ESCs was $98 \%$ after the first and second passages.

\section{AMPK expression in ESCs}

Immunocytochemical and western blotting analyses of AMPK expression were performed to investigate AMPK expression in ESCs, as shown in Fig. 2A. Immunostaining of AMPK was observed in the cytoplasm of both N-ESCs and A-ESCs. A-ESCs exhibited greater AMPK expression
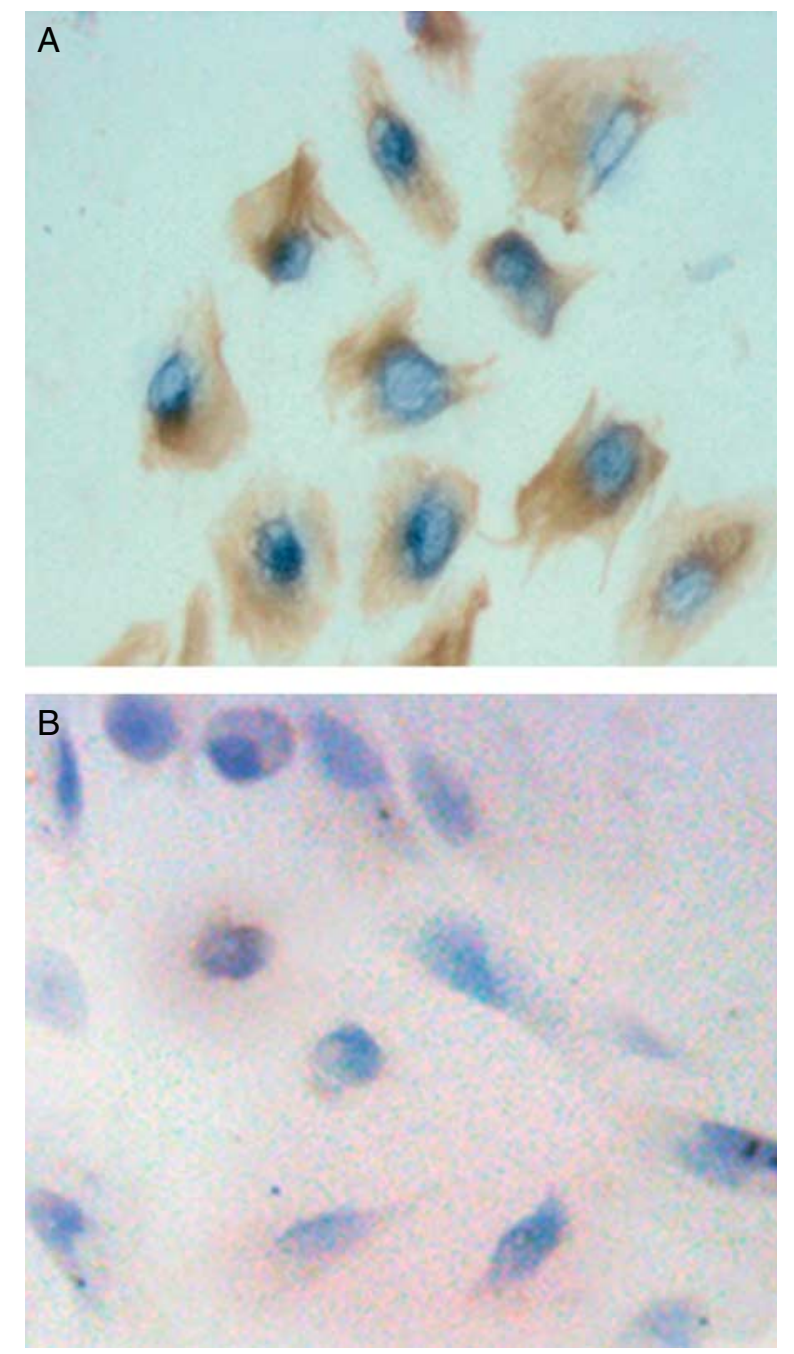

Figure 1 Immunocytochemical staining for vimentin and cytokeratin expression. (A) The cytoplasm of cells was positive for vimentin. The nuclei were stained with hematoxylin. Magnification, $\times 400$. (B) The cytoplasm of cells was immunonegative for cytokeratin. The nuclei were stained with hematoxylin. Magnification, $\times 400$. than N-ESCs $(P<0.05 ;$ Table 1$)$. Eight samples were employed, ESCs isolated from four samples from control group (mean age: $40.5 \pm 2.3$ years) and four from adenomyosis group (mean age: $40.8 \pm 2.3$ years). Figure 2B showed that AMPK expression levels (AMPK/ $\beta$-actin) in A-ESC and N-ESC groups were $83.4 \pm 13.8$ and $58.9 \pm 9.8 \%$ respectively (i.e. 1.4 -fold more).

\section{Compound C inhibited AMPK activation in ESCs}

ESCs isolated from six endometria samples, three from control group (mean age: $42.5 \pm 1.8$ years) and three from adenomyosis group (mean age: $40.8 \pm 2.1$ years), were used in these experiments. Compound $\mathrm{C}$, a selective inhibitor of AMPK, was found to significantly inhibit the phosphorylation of AMPK in a time-dependent manner, in both N-ESCs and A-ESCs (Fig. 3). After $1 \mathrm{~h}$ of exposure, the average inhibition was $2.4 \pm 0.5$-fold in A-ESCs and $2.9 \pm 0.9$-fold in N-ESCs. In both A-ESCs and N-ESCs, there was no difference in the inhibition effect between 1 and $24 \mathrm{~h}$ groups $(P>0.5)$. On the basis of these findings, ESCs pretreated for $1 \mathrm{~h}$ with $40 \mu \mathrm{M}$ compound $\mathrm{C}$ were used in the follow-up experiments.

\section{Metformin inhibits the proliferation of ESCs via AMPK activation}

The same batch cells as was used for the experiments for which the results are illustrated in Fig. 3 were used for MTT assay. The effect of metformin on the proliferation of $\mathrm{N}$-ESCs and A-ESCs were shown in Fig. 4A. Exposure to metformin for $24 \mathrm{~h}$ resulted in marked, concentrationdependent inhibition of growth of both A-ESCs and $\mathrm{N}$-ESCs. The mean $\mathrm{IC}_{50}$ value for A-ESCs was 2.45 and $7.87 \mathrm{mmol} / \mathrm{l}$ for $\mathrm{N}$-ESCs (i.e. threefold less).

A-ESCs isolated from four women (mean age: 40.8 \pm 2.3 years) with metformin were used in the western blot analysis. In the absence of compound C, metformin was associated with a significant $2.0 \pm 0.3$-fold increase in AMPK activation ( $\mathrm{p}$-AMPK/AMPK) compared with controls (Fig. 4B, ${ }^{*} P<0.001$ ), whereas in the presence of compound $C$, this effect was diminished. The relative activated AMPK levels in A-ESCs in control, metformin-, compound $\mathrm{C}_{-}$, and compound $\mathrm{C}+$ metformin-treated groups were $43.9 \pm 1.6,83.2 \pm 6.1,23.2 \pm 5.2$, and $25.1 \pm 4.1 \%$ respectively (Fig. 4 B).

\section{Metformin shows pronounced effects on activation of AMPK signaling in A-ESCs from secretory phase than in cells from proliferative phase}

As shown in Fig. 5A, samples of endometrium were taken from six women, $43.2 \pm 1.5$ years of age, with a diagnosis of adenomyosis, three in secretory phase and three in proliferative phase. Metformin inhibited the growth of A-ESCs from proliferative 

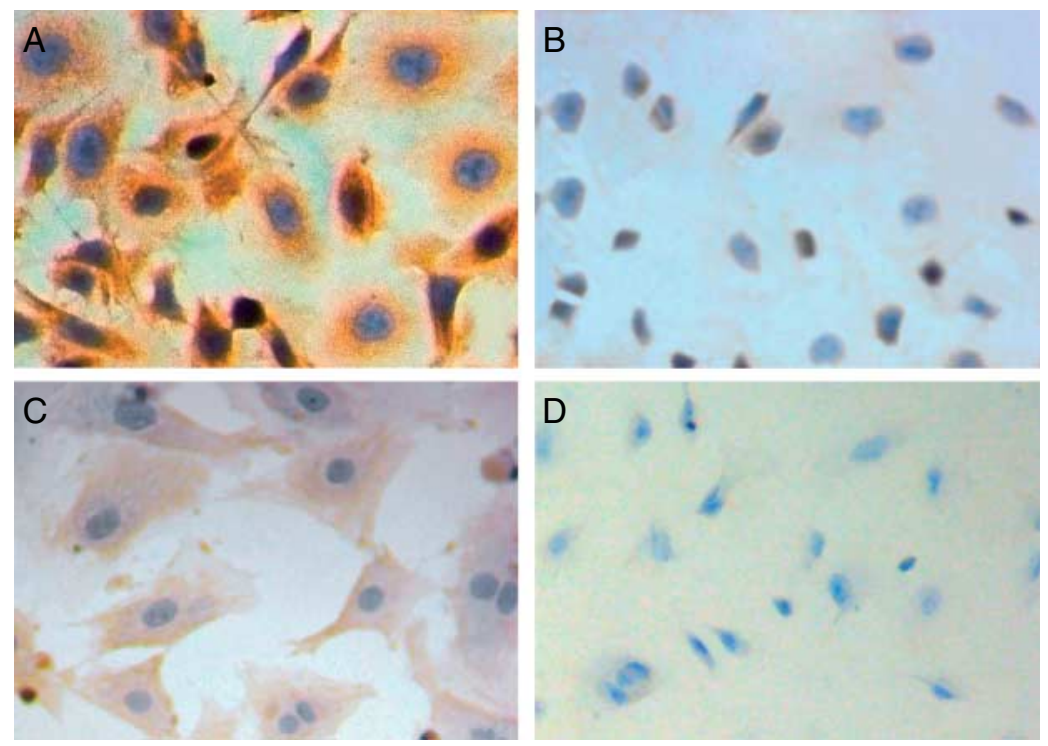

E

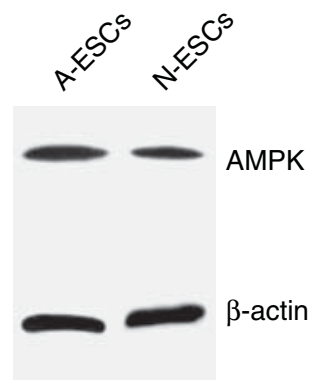

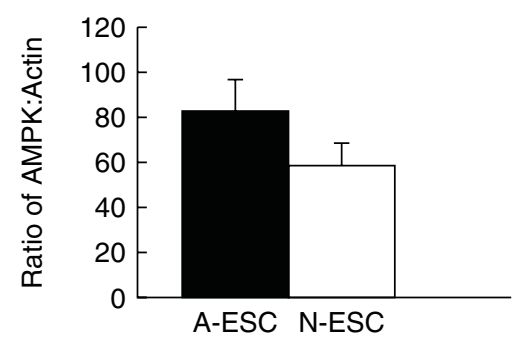

Figure 2 (A) Immunocytochemistry staining for AMPK expression. (a) A-ESCs with AMPK antibody, (b) negative controls for A-ESCs using PBS instead of AMPK as primary antibody, (c) N-ESCs with AMPK antibody, and (d) negative controls for N-ESCs using PBS instead of AMPK as primary antibody. Immunostaining of AMPK was observed in the cytoplasm of both A-ESCs and N-ESCs. The nuclei were stained with hematoxylin. Magnification, $\times 400$. (B) AMPK expression levels (AMPK/ $\beta$-actin) in A-ESCs $(n=4)$ and $\mathrm{N}$-ESCs $(n=4)$. Protein expression was normalized to $\beta$-actin. AMPK expression levels in A-ESC and N-ESC groups were $83.4 \pm 13.8$ and $58.9 \pm 9.8 \%$ respectively (i.e. 1.4-fold more). Data are shown as the median \pm range. phase by $47.8 \pm 1.2 \%$ and cells from secretory phase by $58.0 \pm 0.9 \%$ relative to controls. Metformin-induced inhibition of cell growth was abolished by pretreatment with compound C (40 $\mu \mathrm{M}$ for $1 \mathrm{~h}$ ).

$\mathrm{E}_{2}$ significantly inhibited AMPK activation in both A-ESCs from proliferative phase and cells from secretory phase (Fig. 5B). The same batch cells with $E_{2}$ were used in western blot analysis; the result showed that the average reduction ratio was $2.1 \pm 0.8$-fold in proliferative phase and $2.5 \pm 0.5$-fold in secretory phase.

In order to test our hypothesis, we employed 18 more samples from patients with adenomyosis (mean age: $42.1 \pm 1.8$ years), nine in secretory phase and nine in proliferative phase. The effects of metformin on activation of AMPK signaling differed between the A-ESCs from the proliferative phase and from the secretory phase. As shown in Fig. 5C, in the absence of compound C, the effects of metformin on activation of AMPK signaling (p-AMPK/AMPK) were obvious (Fig. 5C, $* P<0.001$ ). The relative activated AMPK levels in cells during the secretory phase and proliferative phase were $86.8 \pm 4.8$ (2.3-fold increase vs control) and 60.2 \pm 2.5 (1.6-fold increase vs control) respectively. Pretreatment with compound $\mathrm{C}(40 \mu \mathrm{M}$ for $1 \mathrm{~h}$ ) diminished this effect.

\section{AMPK activation inhibits the PI3K/AKT pathway in A-ESCs}

The data presented earlier suggested that metformin inhibited proliferation of A-ESCs through effects on the AMPK pathway. As previous reports revealed, AMPK is upstream of the PI3K/AKT pathway (Hanna et al. 2012). The researchers wanted to know whether AMPK is upstream of the PI3K/AKT pathway in A-ESCs. We used the same-generation of cells that were used for the experiments that yielded the results illustrated in Fig. 5C from 16 women (eight in secretory phase and eight in proliferative phase) in western blot analysis. We investigated the effects of metformin (with or without pretreatment compound $\mathrm{C} 40 \mu \mathrm{M}$ for $1 \mathrm{~h}$ ) on AKT phosphorylation. As illustrated in Fig. 6A and B, the inhibitory effects of metformin on AKT activation

Table 1 Histological score (HSCORE) values of total AMPK expression in A-ESCs and N-ESCs.

\begin{tabular}{lcc}
\hline & No. of slides & HSCORE $($ mean \pm s.D. $)$ \\
\hline A-ESCs & 30 & $3.4 \pm 0.1$ \\
N-ESCs & 17 & $2.2 \pm 0.2$ \\
\hline
\end{tabular}

$P<0.05$, A-ESCs vs N-ESCs. 


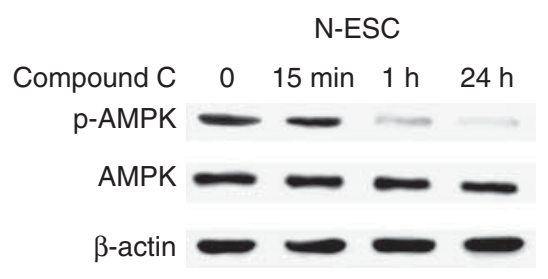

A-ESC

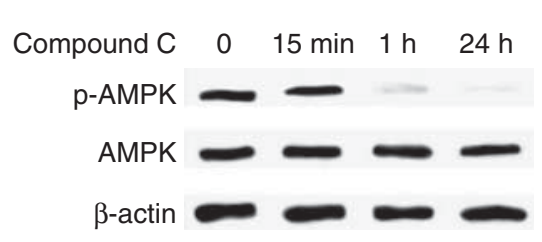

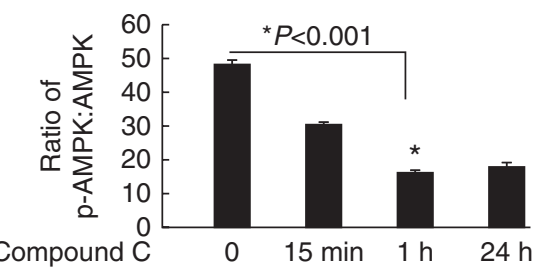

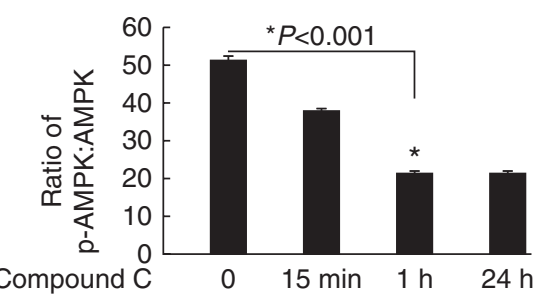

Figure 3 Compound $\mathrm{C}$ inhibited AMPK activation in ESCs. Compound C was found to significantly inhibit the phosphorylation of AMPK in a timedependent manner, in both N-ESCs $(n=3)$ and A-ESCs $(n=3)$. In the A-ESC group, the ratio of p-AMPK:AMPK was $21.15 \pm 1.2 \%$ at $1 \mathrm{~h}$ and $21.53 \pm 0.1 \%$ at $24 \mathrm{~h}$. In the N-ESC group, the ratio was $16.06 \pm 0.7$ and $18.06 \pm 1.4 \%$ respectively. After $1 \mathrm{~h}$ of exposure, the average inhibition was $2.4 \pm 0.5$-fold in A-ESCs and 2.9 \pm 0.9 -fold in $\mathrm{N}$-ESCs. In both A-ESCs and N-ESCs, there was no difference regarding the inhibitory effect between 1 and $24 \mathrm{~h}$ groups $(P>0.5)$. The concentration of $\mathrm{CC}$ is $40 \mu \mathrm{mol} / \mathrm{l}, * P<0.001$ vs control group. Data are shown as the median \pm range.
(p-AKT/AKT) were more pronounced in A-ESCs from the secretory phase (3.2-fold inhibition vs control) than in those from the proliferation phase (2.3-fold inhibition vs control). The inhibitory effect of AKT phosphorylation was abolished by compound C pretreatment (Fig. 6A and B, $P<0.001$ ). The results showed that PI3K/AKT pathway can be inhibited by upregulating AMPK phosphorylation with metformin.

\section{Discussion}

Adenomyosis has been described as an 'elusive disease' or 'enigma' (Thomas \& Clark 1989), and there is a genuine need for new chemotherapeutic strategies to treat it. Adenomyosis results from the growth of ectopic endometrium, a process that requires coordination of cellular energy status (Osteen et al. 2002). In a previous study, researchers showed that activation of the energy-sensing AMPK pathway can inhibit cell proliferation in lung cancer cells (Su et al. 2010). In this study, AMPK expression level is higher in A-ECSs than in $\mathrm{N}$-ESCs. On the basis of these discoveries, we considered it worthwhile to investigate AMPK as a possible target for therapeutic intervention in adenomyosis.

AMPK expression has been previously studied in other tissues, such as hippocampal neurons and fibroblasts (Amato et al. 2011), but, to our knowledge, no report about AMPK expression in ESCs has been published. In this study, we used immunostaining and western blot analysis to evaluate the expression levels of AMPK in $\mathrm{N}$-ESCs and A-ESCs. Our results indicated that AMPK expression was higher in the cytoplasm of A-ESCs than in that of N-ESCs. This indicates that if we find drugs with an effect on the AMPK signaling pathway this might lead to breakthroughs in the treatment of adenomyosis.

A

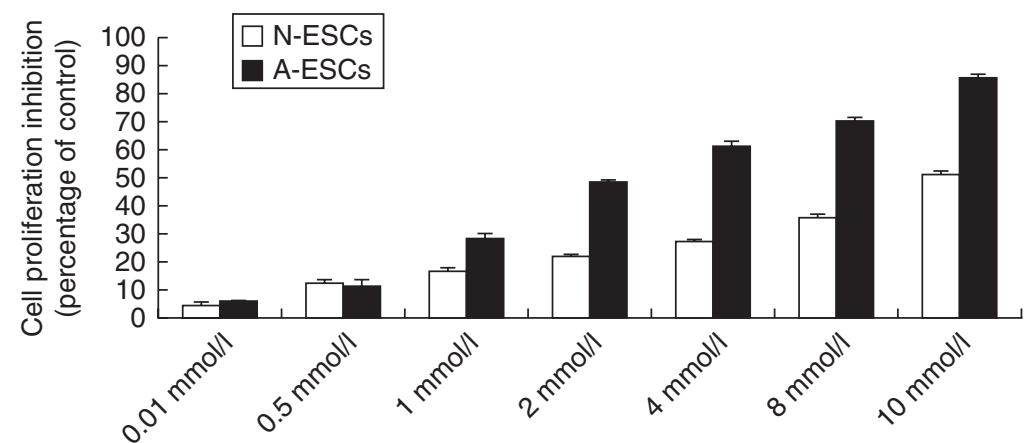

B

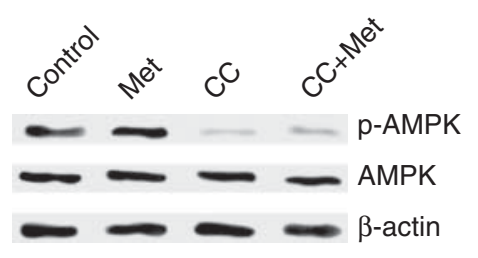

Reproduction (2013) 146 397-406
Figure 4 (A) Met inhibited proliferation of ESCs. In both N-ESCs $(n=3)$ and A-ESCs $(n=3)$, and Met $(0.01-10 \mathrm{mmol} / \mathrm{l})$ inhibited cell proliferation in a concentration-dependent manner. Met was found to be more effective in A-ESCs $\left(\mathrm{IC}_{50} 2.45 \mathrm{mmol} / \mathrm{l}\right)$ than in $\mathrm{N}$-ESCs ( $\left.\mathrm{IC}_{50} 7.87 \mathrm{mmol} / \mathrm{l}\right)$ (i.e. threefold less). (B) Met increased AMPK activation in A-ESCs. A-ESCs were incubated for $1 \mathrm{~h}$ with or without $40 \mu \mathrm{mol} / \mathrm{l}$ of CC prior to stimulation with $2 \mathrm{mmol} / \mathrm{l}$ Met for $24 \mathrm{~h}$. Protein expression was normalized to $\beta$-actin. In the absence of CC, Met was associated with a significant 2.0 \pm 0.3 -fold increase in AMPK activation (p-AMPK/AMPK) compared with controls $(* P<0.001)$, whereas in the presence of $C C$, this effect was diminished. The relative activated AMPK levels in A-ESCs in control, Met-, CC-, and CC+Met-treated groups were $43.9 \pm 1.6 \%, 83.2 \pm 6.1 \%, 23.2 \pm 5.2 \%$, and $25.1 \pm 4.1 \%$ respectively, $n=4$. Met, metformin; CC, compound C. Data are shown as the median \pm range. 


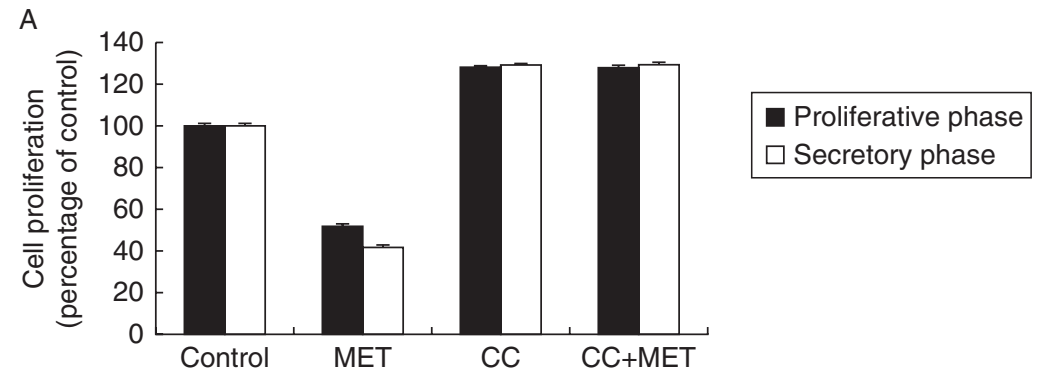

в
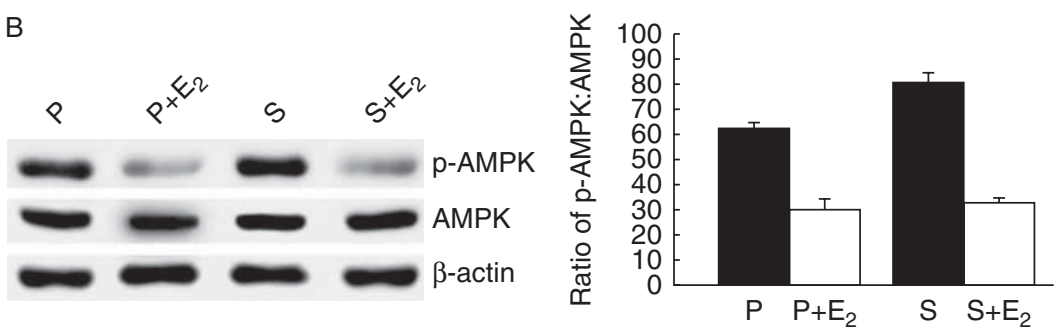

c
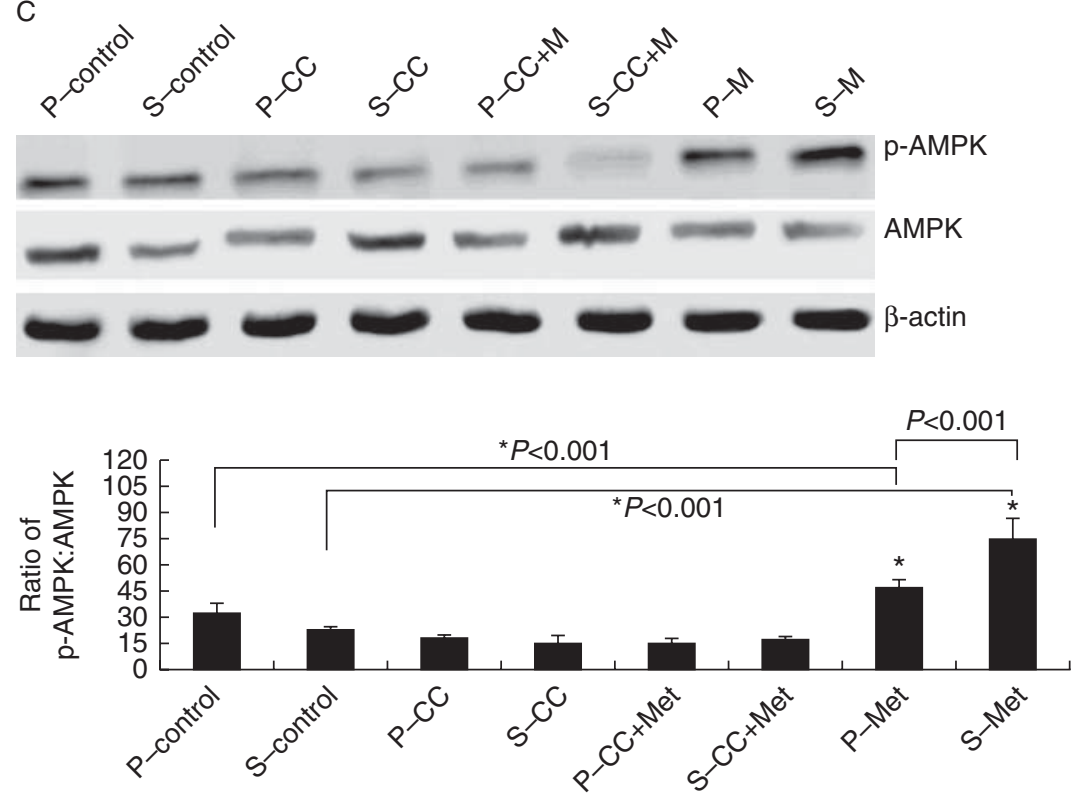

Figure 5 (A) Met inhibition of the growth of A-ESCs alters with the menstrual cycle. A-ESCs were incubated for $1 \mathrm{~h}$ with or without $40 \mu \mathrm{mol} / \mathrm{I}$ of CC prior to stimulation with $2 \mathrm{mmol} / \mathrm{l}$ Met for $24 \mathrm{~h}$. A-ESC growth was assessed by MTT assay. Met inhibited growth of A-ESCs from the proliferative phase $(n=3)$ by $52.2 \pm 1.2 \%$ and of cells from the secretory phase $(n=3)$ by $42.0 \pm 0.9 \%$ relative to controls. (B) $E_{2}$ significantly inhibited AMPK activation. A-ESCs from the proliferative phase $(n=3)$ and from the secretory phase $(n=3)$ were incubated with or without $10 \mathrm{nM} \mathrm{E}_{2}$ for $24 \mathrm{~h}$. Protein expression was normalized to $\beta$-actin. The average reduction ratio was $2.1 \pm 0.8$-fold in the proliferative phase and $2.5 \pm 0.5$-fold in the secretory phase.

(C) The effect of Met on activation of AMPK signaling was more pronounced in A-ESCs from the secretory phase. A-ESCs were incubated for $1 \mathrm{~h}$ with or without $40 \mu \mathrm{M} \mathrm{CC}$ prior to stimulation with $2 \mathrm{mmol} / \mathrm{l}$ Met for $24 \mathrm{~h}$. Protein expression was normalized to $\beta$-actin. $S$, secretory phase $(n=9) ; P$, proliferative phase $(n=9)$. ${ }^{*} P<0.001$ vs control group; $P<0.001$ secretory phase + Met vs proliferative phase + Met. Met, metformin; CC, compound C. Data are expressed as mean \pm S.E.M.
Metformin, a biguanide drug widely used for the treatment of type 2 diabetes (Radziuk et al. 2003), is considered as an insulin sensitizer (Polyzos et al. 2010). Metformin also has beneficial effects on endometrial abnormalities, such as hyperplasia and cancerous lesions (Palomba et al. 2009). Expression of the insulin receptor gene is regulated during the menstrual cycle, enabling insulin to inhibit stromal cell decidualization (Lockwood et al. 2007). Recent evidence suggested that metformin inhibits hepatic gluconeogenesis by inhibiting mitochondria complex I activity (Owen et al. 2000) and activating its downstream target, AMPK, which can regulate multiple signaling pathways controlling cell proliferation.

We found that metformin was associated with a significant increase in AMPK activation. The effects of metformin on activation of AMPK signaling and inhibition of A-ESC proliferation were more pronounced during the secretory phase, suggesting that application of metformin to patients with adenomyosis during secretory phase would be more effective.

Metformin has been the agent most frequently studied in patients with polycystic ovarian syndrome (Cheang \& Nestler 2004). It has been suggested that metformin may reduce the risk of first-trimester miscarriage (Glueck et al. 2001). Meta-analysis supports the hypothesis that metformin is beneficial in improving clinical pregnancy and ovulation rates (Tang et al. 2010). In our study, the inhibitory effect by metformin on endometrial cells was dose dependent. The concentration of metformin required for inhibition of cell proliferation $(2 \mathrm{mM})$ was much higher than the doses used clinically 

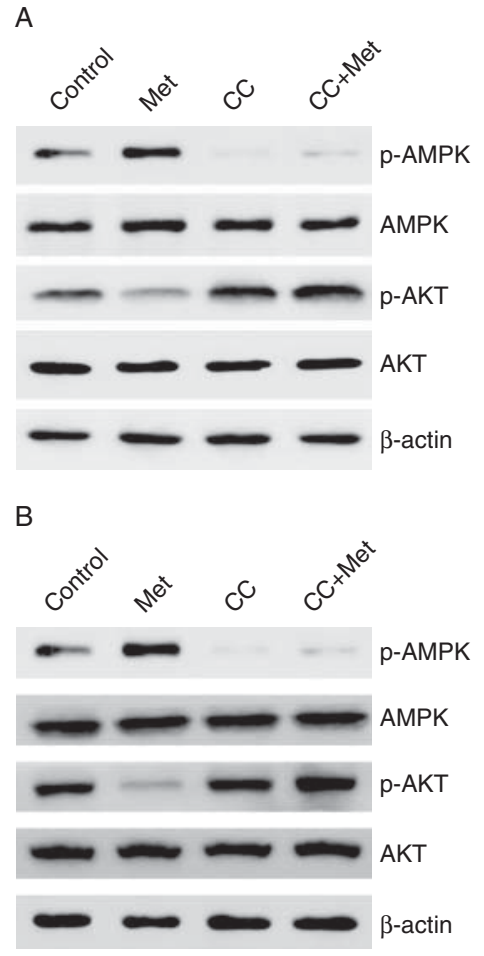
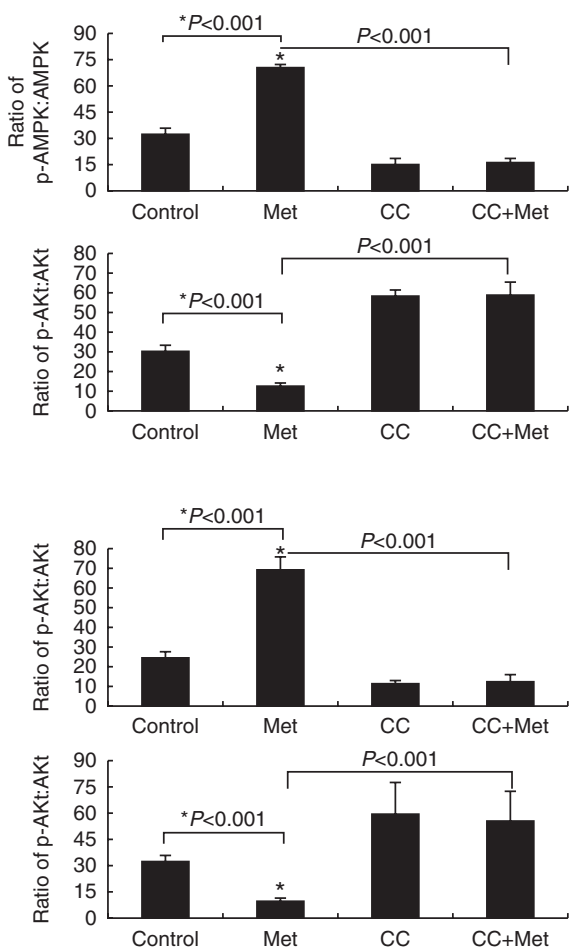

Figure 6 AMPK activation inhibited AKT signaling in A-ESCs. (A) A-ESCs from proliferative phase, ${ }^{*} P<0.001$ vs control group $(n=8)$. (B) A-ESCs from secretory phase, $* P<0.001$ vs control group $(n=8)$. A-ESCs were incubated for $1 \mathrm{~h}$ with or without $40 \mu \mathrm{mol} / \mathrm{l} \mathrm{CC}$ prior to stimulation with $2 \mathrm{mmol} / \mathrm{l}$ Met for $24 \mathrm{~h}$. Protein expression was normalized to $\beta$-actin. The inhibitory effects of Met on p-AKT/AKT were 3.2-fold inhibition in the secretory phase vs control and 2.3 -fold inhibition in the proliferative phase. The inhibitory effect of AKT phosphorylation was abolished by CC pretreatment $(P<0.001)$. Met, metformin; CC, compound C. Data are expressed as the mean \pm S.E.M. (i.e. $0.465-2.5 \mathrm{mg} / \mathrm{l}$; Al-Jebawi et al. 1998). The very large doses of metformin used in our in vitro cultures cannot be replicated clinically. Further research is needed using our data to search for other compounds that increase AMPK activation more effectively for treatment of adenomyosis.

Interestingly, adenomyosis has been reported to be associated with enhanced expression of nerve growth factor $\beta$ (Li et al. 2011), tyrosine kinase receptor B (Huang et al. 2011), and PAK1 (Kim et al. 2010), all of which are thought to signal through PI3K. PI3K/AKT signaling plays an important role in cell survival. It is aberrantly activated in adenomyosis (Zhang et al. 2010). Previous studies showed that AMPK acts upstream of the PI3K/AKT pathway in hippocampal neurons and fibroblasts (Amato et al. 2011). Here, we found that metformin can increase AMPK phosphorylation and decrease AKT phosphorylation in A-ESCs. An AMPK inhibitor can inhibit AMPK phosphorylation and increase AKT phosphorylation in A-ESCs. These findings indicate a functional interaction between the AMPK and PI3K/AKT pathways in A-ESCs, with AMPK activation leading to inhibition of AKT signaling. AKT phosphorylation was negatively correlated with AMPK activation in A-ESCs. So in A-ESCs, AMPK also acts upstream of the PI3K/AKT pathway. Also, metformin acts as a novel AKT inhibitor and can be used to inhibit the proliferation of human A-ESCs.

Consistent with our findings, metformin has been reported to be as effective as letrozole (an aromatase inhibitor that reduces estrogen levels) in inducing regression of endometriotic tissue in a rat model of endometriosis (Oner et al. 2010). It has also been shown to repress tumor growth in xenograft tumor models of breast, prostate, and colon cancers (Belda-Iniesta et al. 2011). Recent epidemiological evidence suggests that metformin can lower the risk of cancer and reduce cancer incidence and cancer-related deaths among diabetic patients (Decensi et al. 2010). Furthermore, a retrospective cohort study of patients with early-stage endometrial cancer showed that patients receiving metformin and adjuvant chemotherapy had a higher response rate than those receiving adjuvant chemotherapy alone (Jalving et al. 2010). These findings have prompted further research into the potential role of metformin in cancer treatment and prevention (Ben Sahra et al. 2010).

In conclusion, our study showed that metformin can inhibit the proliferation of A-ESCs through activation of AMPK and inhibition of the PI3K/AKT signaling pathway. This study indicates that metformin may be an option for treatment of adenomyosis and needs further investigation.

\section{Declaration of interest}

The authors declare that there is no conflict of interest that could be perceived as prejudicing the impartiality of the research reported.

\section{Funding}

This study was supported by grants from the Foundation for Excellent Young and Middle-Aged Scientists of Shandong Province (no. 2008BS03040) and the Natural Science Foundation of Shandong Province (no. ZR2011HQ046). 


\section{References}

Al-Jebawi AF, Lassman MN \& Abourizk NN 1998 Lactic acidosis with therapeutic metformin blood level in a low-risk diabetic patient. Diabetes Care 21 1364-1365. (doi:10.2337/diacare.21.8.1364)

Amato S, Liu X, Zheng B, Cantley L, Rakic P \& Man HY 2011 AMP-activated protein kinase regulates neuronal polarization by interfering with $\mathrm{PI}$ 3-kinase localization. Science 332 247-251. (doi:10.1126/science. 1201678)

Ascher SM, Arnold LL, Patt RH, Schruefer JJ, Bagley AS, Semelka RC, Zeman RK \& Simon JA 1994 Adenomyosis: prospective comparison of MR imaging and transvaginal sonography. Radiology 190 803-806.

Atri M, Reinhold C, Mehio AR, Chapman WB \& Bret PM 2000 Adenomyosis: US features with histologic correlation in an in-vitro study. Radiology 215 783-790.

Bazot M, Cortez A, Darai E, Rouger J, Chopier J, Antoine JM \& Uzan S 2001 Ultrasonography compared with magnetic resonance imaging for the diagnosis of adenomyosis: correlation with histopathology. Human Reproduction 16 2427-2433. (doi:10.1093/humrep/16.11.2427)

Belda-Iniesta C, Pernía O \& Simó R 2011 Metformin: a new option in cancer treatment. Clinical \& Translational Oncology 13 363-367. (doi:10.1007/s12094-011-0669-y)

Ben Sahra I, Le Marchand-Brustel Y, Tanti JF \& Bost F 2010 Metformin in cancer therapy: a new perspective for an old antidiabetic drug? Molecular Cancer Therapeutics 9 1092-1099. (doi:10.1158/15357163.MCT-09-1186)

Brown HK, Stoll BS, Nicosia SV, Fiorica JV, Hambley PS, Clarke LP \& Silbiger ML 1991 Uterine junctional zone: correlation between histologic findings and MR imaging. Radiology 179 409-413.

Bruse C, Guan Y, Carlberg M, Carlström K \& Bergqvist A 2005 Basal release of urokinase plasminogen activator, plasminogen activator inhibitor-1, and soluble plasminogen activator receptor from separated and cultured endometriotic and endometrial stromal and epithelial cells. Fertility and Sterility 83 (Suppl 1) 1155-1160. (doi:10.1016/j.fertnstert. 2004.09.033)

Cakmak H, Guzeloglu-Kayisli O, Kayisli UA \& Arici A 2009 Immuneendocrine interactions in endometriosis. Frontiers in Bioscience $1429-443$.

Cantrell LA, Zhou C, Mendivil A, Malloy KM, Gehrig PA \& Bae-Jump VL 2010 Metformin is a potent inhibitor of endometrial cancer cell proliferation - implications for a novel treatment strategy. Gynecologic Oncology 116 92-98. (doi:10.1016/j.ygyno.2009.09.024)

Carling D 2004 The AMP-activated protein kinase cascade - a unifying system for energy control. Trends in Biochemical Sciences 29 18-24. (doi:10.1016/j.tibs.2003.11.005)

Carling D, Thornton C, Woods A \& Sanders MJ 2012 AMP-activated protein kinase: new regulation, new roles? Biochemical Journal 445 11-27. (doi:10.1042/BJ20120546)

Cheang KI \& Nestler JE 2004 Should insulin-sensitizing drugs be used in the treatment of polycystic ovary syndrome? Reproductive BioMedicine Online 8 440-447. (doi:10.1016/S1472-6483(10)60928-5)

Chen S, Rehman SK, Zhang W, Wen A, Yao L \& Zhang J 2010 Autophagy is a therapeutic target in anticancer drug resistance. Biochimica et Biophysica Acta 1806 220-229. (doi:10.1016/j.bbcan. 2010.07.003)

Decensi A, Puntoni M, Goodwin P, Cazzaniga M, Gennari A, Bonanni B \& Gandini S 2010 Metformin and cancer risk in diabetic patients: a systematic review and meta-analysis. Cancer Prevention Research 3 1451-1461. (doi:10.1158/1940-6207.CAPR-10-0157)

Devlieger R, D'Hooghe T \& Timmerman D 2003 Uterine adenomyosis in the infertility clinic. Human Reproduction Update 9 139-147. (doi:10.1093/humupd/dmg010)

Dueholm M, Lundorf E, Hansen ES, Sorensen JS, Ledertoug S \& Olesen F 2001 Magnetic resonance imaging and transvaginal ultrasonography for the diagnosis of adenomyosis. Fertility and Sterility 76 588-594. (doi:10.1016/S0015-0282(01)01962-8)

Glueck CJ, Phillips H, Cameron D, Sieve-Smith L \& Wang P 2001 Continuing metformin throughout pregnancy in women with polycystic ovary syndrome appears to safely reduce first-trimester spontaneous abortion: a pilot study. Fertility and Sterility 75 46-52. (doi:10.1016/ S0015-0282(00)01666-6)

Hanna RK, Zhou C, Malloy KM, Sun L, Zhong Y, Gehrig PA \& Bae-Jump VL 2012 Metformin potentiates the effects of paclitaxel in endometrial cancer cells through inhibition of cell proliferation and modulation of the mTOR pathway. Gynecologic Oncology 125 458-469. (doi:10.1016/ j.ygyno.2012.01.009)

Hardie DG 2011 AMP-activated protein kinase: an energy sensor that regulates all aspects of cell function. Genes and Development 25 1895-1908. (doi:10.1101/gad.17420111)

Harvey J \& Warwick I 2010 Endometriosis. BMJ 340 c2661. (doi:10.1136/ bmj.c2661)

Huang Y, Zheng W, Mu L, Ren Y, Chen X \& Liu F 2011 Expression of tyrosine kinase receptor $B$ in eutopic endometrium of women with adenomyosis. Archives of Gynecology and Obstetrics 283 775-780. (doi:10.1007/ s00404-010-1718-4)

Jalving M, Gietema JA, Lefrandt JD, de Jong S, Reyners AK, Gans RO \& de Vries EG 2010 Metformin: taking away the candy for cancer? European Journal of Cancer 46 2369-2380. (doi:10.1016/j.ejca.2010. 06.012)

Kim SR, Kim SH, Lee HW, Chae HD, Kim CH \& Kang BM 2010 Increased expression of p21-activated kinase in adenomyosis. Fertility and Sterility 94 1125-1128. (doi:10.1016/j.fertnstert.2009.11.048)

Leyendecker G, Wildt L \& Mall G 2009 The pathophysiology of endometriosis and adenomyosis: tissue injury and repair. Archives of Gynecology and Obstetrics 280 529-538. (doi:10.1007/s00404-0091191-0)

Li Y, Zhang SF, Zou SE, Xia X \& Bao L 2011 Accumulation of nerve growth factor and its receptors in the uterus and dorsal root ganglia in a mouse model of adenomyosis. Reproductive Biology and Endocrinology 930. (doi:10.1186/1477-7827-9-30)

Lim CT, Kola B \& Korbonits M 2009 AMPK as a mediator of hormonal signalling. Journal of Molecular Endocrinology 44 87-97. (doi:10.1677/ JME-09-0063)

Lockwood CJ, Krikun G, Rahman M, Caze R, Buchwalder L \& Schatz F 2007 The role of decidualization in regulating endometrial hemostasis during the menstrual cycle, gestation, and in pathological states. Seminars in Thrombosis and Hemostasis 33 111-117. (doi:10.1055/s2006-958469)

Makker A, Goel MM, Das V \& Agarwal A 2012 PI3K-Akt-mTOR and MAPK signaling pathways in polycystic ovarian syndrome, uterine leiomyomas and endometriosis: an update. Gynecological Endocrinology 28 175-181. (doi:10.3109/09513590.2011.583955)

Martelli AM, Chiarini F, Evangelisti C, Ognibene A, Bressanin D, Billi AM, Manzoli L, Cappellini A \& McCubrey JA 2012 Targeting the liver kinase B1/AMP-activated protein kinase pathway as a therapeutic strategy for hematological malignancies. Expert Opinion on Therapeutic Targets 16 729-742. (doi:10.1517/14728222.2012.694869)

Noyes RW, Hertig AT \& Rock J 1975 Dating the endometrial biopsy. American Journal of Obstetrics and Gynecology 122 262-263.

Oner G, Ozcelik B, Ozgun MT, Serin IS, Ozturk F \& Basbug M 2010 The effects of metformin and letrozole on endometriosis and comparison of the two treatment agents in a rat model. Human Reproduction $\mathbf{2 5}$ 932-937. (doi:10.1093/humrep/deq016)

Osteen KG, Bruner-Tran KL, Keller NR \& Eisenberg E 2002 Progesteronemediated endometrial maturation limits matrix metalloproteinase (MMP) expression in an inflammatory-like environment: a regulatory system altered in endometriosis. Annals of the New York Academy of Sciences 955 37-47 (discussion 86-38, 396-406). (doi:10.1111/j.1749-6632. 2002.tb02764.x)

Owen MR, Doran E \& Halestrap AP 2000 Evidence that metformin exerts its anti-diabetic effects through inhibition of complex 1 of the mitochondrial respiratory chain. Biochemical Journal 348 607-614. (doi:10.1042/ 0264-6021:3480607)

Palomba S, Falbo A, Zullo F \& Orio F Jr 2009 Evidence-based and potential benefits of metformin in the polycystic ovary syndrome: a comprehensive review. Endocrine Reviews 30 1-50. (doi:10.1210/er.2008-0030)

Polyzos SA, Kountouras J, Zavos C \& Tsiaousi E 2010 The role of adiponectin in the pathogenesis and treatment of non-alcoholic fatty liver disease. Diabetes, Obesity \& Metabolism 12 365-383. (doi:10.1111/j.1463-1326.2009.01176.x)

Radziuk J, Bailey CJ, Wiernsperger NF \& Yudkin JS 2003 Metformin and its liver targets in the treatment of type 2 diabetes. Current Drug Targets. Immune, Endocrine and Metabolic Disorders 3 151-169. (doi:10.2174/ 1568008033340298) 
Reinhold C, McCarthy S, Bret PM, Mehio A, Atri M, Zakarian R, Glaude Y, Liang L \& Seymour RJ 1996 Diffuse adenomyosis: comparison of endovaginal US and MR imaging with histopathologic correlation. Radiology 199 151-158.

Su MY, Hsieh SY, Lee YR, Chang MC, Yuan TT \& Chang JM 2010 The relationship between energy status and AMP-activated protein kinase in human $\mathrm{H} 460$ lung cancer cells. Cell Biochemistry and Function 28 549-554. (doi:10.1002/cbf.1685)

Sugawara J, Fukaya T, Murakami T, Yoshida H \& Yajima A 1997 Increased secretion of hepatocyte growth factor by eutopic endometrial stromal cells in women with endometriosis. Fertility and Sterility 68 468-472. (doi:10.1016/S0015-0282(97)00226-4)

Takeuchi M \& Matsuzaki K 2011 Adenomyosis: usual and unusual imaging manifestations, pitfalls, and problem-solving MR imaging techniques. Radiographics 31 99-115. (doi:10.1148/rg.311105110)

Tang T, Lord JM, Norman RJ, Yasmin E \& Balen AH 2010 Insulin-sensitising drugs (metformin, rosiglitazone, pioglitazone, D-chiro-inositol) for women with polycystic ovary syndrome, oligo amenorrhoea and subfertility. Cochrane Database of Systematic Reviews. Issue 4 CD003053. (doi:10.1002/14651858.CD003053.pub3)

Tao R, Gong J, Luo X, Zang M, Guo W, Wen R \& Luo Z 2010 AMPK exerts dual regulatory effects on the PI3K pathway. Journal of Molecular Signaling 5 1. (doi:10.1186/1750-2187-5-1)

Thomas JS Jr \& Clark JF 1989 Clark adenomyosis: a retrospective view. Journal of the National Medical Association 81 969-972.
Towler MC \& Hardie DG 2007 AMP-activated protein kinase in metabolic control and insulin signaling. Circulation Research 100 328-341. (doi:10.1161/01.RES.0000256090.42690.05)

van Veelen W, Korsse SE, van de Laar L \& Peppelenbosch MP 2011 The long and winding road to rational treatment of cancer associated with LKB1/AMPK/TSC/mTORC1 signaling. Oncogene 30 2289-2303. (doi:10.1038/onc.2010.630)

Yang JH, Wu MY, Chen CD, Chen MJ, Yang YS \& Ho HN 2007 Altered apoptosis and proliferation in endometrial stromal cells of women with adenomyosis. Human Reproduction 22 945-952. (doi:10.1093/humrep/ del493)

Zhang H, Zhao X, Liu S, Li J, Wen Z \& Li M $201017 \beta E_{2}$ promotes cell proliferation in endometriosis by decreasing PTEN via NFKB-dependent pathway. Molecular and Cellular Endocrinology 317 31-43. (doi:10.1016/ j.mce.2009.11.009)

Received 30 March 2013

First decision 20 May 2013

Revised manuscript received 24 July 2013

Accepted 31 July 2013 